\section{Adherens junctions: which way is up?}

Epithelial cells connect to each other through specialized structures called adherens junctions, which contain multiprotein complexes that dynamically interact with actin filaments to stabilize the formation and location of these junctions. A recent study by Pilot et al. (Nature, 423, $580-584 ; 2006$ ) has now identified a new pathway that organizes actin filaments at adherens junctions in embryonic epithelia.

To identify components involved in the formation of adherens junctions in primary embryonic epithelia, where this process is ill-understood, Pilot et al. performed microarray-based RNA interference (RNAi) analysis in Drosophila embryos and identified bitesize (btsz), the only Drosophila member of the synaptotagmin-like protein family, as a regulator of embryonic epithelial integrity. Time lapse microscopy of embryos depleted for Btsz indicated that although targeting of key components of adherens junctions was normal, the structures failed to stabilize properly. The authors found that the Btsz2 isoform is apically localized in wild-type embryos and that its localization is dependent on Bazooka (Baz), a protein known to be important for adherens junction formation. Btsz2 cans also bind PtdIns $(4,5) \mathrm{P}_{2}$, a plasma membrane lipid. Inhibiting PtdIns $(4,5) \mathrm{P}_{2}$ not only resulted in epithelial defects similar to btsz mutants, but also defective recruitment of Btsz to the plasma membrane. The authors also found that $\operatorname{PtdIns}(4,5) \mathrm{P}_{2}$ localization is polarized in the embryonic epitheial - it is enriched in the apical-lateral region. Thus, both PtdIns $(4,5) \mathrm{P}_{2}$ and Baz act as upstream polarity cues to localize Btsz and organize actin at the apical junctional region (AJR).

The group went on to examine how Btsz organizes F-actin in the AJR (see figure). Moesin, the only Drosophila F-actin-binding protein that

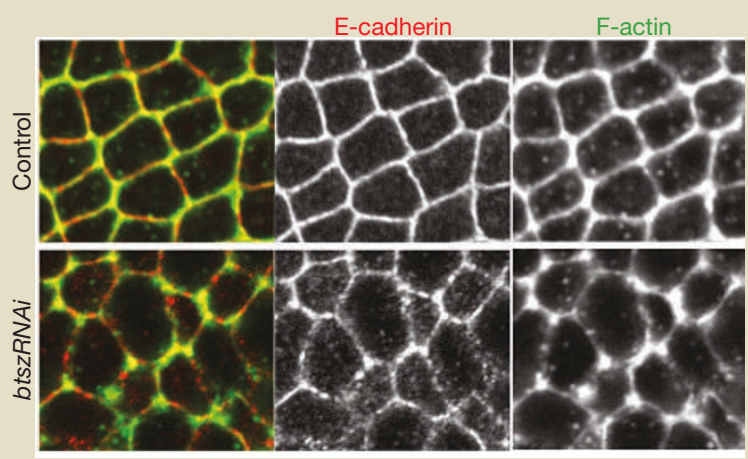

Loss of btsz produces a destabilization of E-cadherin (red) and its heterogenous distriution during junction collapse. Distribution of F-actin (green), is also affected in btszRNAi embryos.

has been implicated in epithelial polarity, had previously been identified as interacting with Btsz in a two-hybrid screen. The authors defined a minimal region of Btsz that interacts with Moesin and showed that both proteins localize to the apical surface and in the AJR, suggesting that this interaction spatially defines a domain for actin organization in the AJR. In btsz mutant embryos, Moesin localization in the AJR was diminished. The authors provide genetic data demonstrating that perturbing Moesin function results in epithelial defects similar to those observed in btsz mutants, suggesting that adherens junctions must be stabilized by the actin cytoskeleton for function.

This study begins to explain the underlying spatial control of actin filaments in the stability of adherens junctions. Mammalian cells contain several close orthologues of Btsz and future investigations will surely address whether they have a conserved function in organizing adherens junctions.

SINÉAD HAYES 